\title{
Better Together: Immunotherapy as Future Combination Strategy for Breast Cancer
}

\author{
Elisa Zanardi ${ }^{1^{*}}$ and Serena Di Cosimo ${ }^{2}$ \\ ${ }^{1}$ Academic Unit of Medical Oncology, Department of Internal Medicine (Di.M.I.), School of Medicine, University of Genoa, Italy \\ ${ }^{2}$ Department of Medical Oncology, Fondazione IRCCS Istituto Nazionale dei Tumori, Milan, Italy
}

"Corresponding author: Elisa Zanardi, Academic Unit of Medical Oncology, Department of Internal Medicine (Di.M.I.), School of Medicine, University of Genoa, Largo Rosanna Benzi 10, Genoa, Italy, Tel: 39 0105600503; Fax: 39 010352753; E-mail: elisa.zanardi@unige.it

Received date: May 04, 2016, Accepted date: June 07, 2016, Published date: June 13, 2016

Copyright: $\odot 2016$ Zanardi E, et al. This is an open-access article distributed under the terms of the Creative Commons Attribution License, which permits unrestricted use, distribution, and reproduction in any medium, provided the original author and source are credited.

\section{Introduction}

In our recent review we described the deep change obtained in the management of breast cancer (BC) over the last decade using combinations of different types of treatment [1]. Better knowledge of oncogenesis, biological characteristics of different types of cancer and the introduction of new drugs have identified possible combinations to overcome the multiple mechanisms of resistance put in place by tumor cells to achieve proliferation and progression.

Given the important and increasingly encouraging results obtained with immunological therapies, particularly in the treatment of melanoma [2-6], non-small cell lung carcinoma (NSCLC) [7], and renal cell carcinoma (RCC) [8], we considered necessary to contribute with a brief comment about these new therapeutic approaches and their possible role in the management of $\mathrm{BC}$, particularly as a combination strategy.

The understanding of the so called "immune checkpoints", capable of regulating T-cell suppression, has led to the development of immune checkpoint inhibitors for cancer treatment. Nowadays the better known immune checkpoint inhibitors are represented by the blocking antibody $(\mathrm{Ab})$ against T-Lymphocyte-associated-protein-4 (CTLA-4), the Abs against programmed death-1 (PD-1) and the $\mathrm{Ab}$ against programmed death-ligand-1 (PD-L1).

CTLA-4 expression is induced in activated T-cell, it competes with CD28 receptor binding CD80 and CD86 with approximately 100 -fold greater affinity than the CD28 receptor, and leads to a reduction of Tcell population therefore; CTLA-4 downregulates T-cell function. As a confirmation of the T-cells function suppression induced by CTLA-4, mice deficient in CTLA-4 die from fatal lymphoproliferation $[9,10]$. The inhibition of CTLA-4 involves the loss of control on T-cells determining cancer cells death and antitumor immunity. Ipilimumab (Yervoy, Bristol Myers Squibb, Princeton, NJ) was the first antiCTLA- 4 Ab clinically approved after the milestone trials that showed a significant improvement in overall survival (OS) for patients with metastatic melanoma $[2,3]$.

PD-1 is expressed on antigen-experienced memory T-cells in peripheral tissues; when PD- 1 is engaged by its ligands, PD-L1 and PD-L2, it inhibits T-cell activation thus resulting in a negative regulation of T-cell activity. PD-1 is also expressed on B-cells, monocytes, dendritic cells and NK cells therefore, blockade of the PD-1 pathway may influence the function of these cell as well [11]. The preclinical rationale that inhibition of PD-1 could lead to antitumor activity mediated by the immune system was confirmed by the phase III clinical trials in different solid tumors such as melanoma, NSCLC and RCC. Nivolumab (Opdivo', Bristol-Myers Squibb, Princeton, NJ) is an IgG4 fully human anti-PD-1 Ab recently approved for the treatment of metastatic melanoma, NSCLC and RCC $[5,7,8]$, and Pembrolizumab (Keytruda', Merck, Darmstadt, Germany) is an IgG4 engineered humanized anti-PD-1 Ab approved for the treatment of metastatic melanoma and NCSLC $[6,12]$.

PD-L1 is expressed on many cell types, including T-cells, B-cells, monocytes, antigen-presenting cell and epithelial cells. PD-L1 is believed to exert negative signals on T-cells not only by binding PD-1 but also by interacting with B7, expressed on dendritic cells [13]. Atezolizumab (MPDL3280A, Roche) is an IgG4 engineered fully human anti-PD-L1 Ab that doubled OS compared with docetaxel in previously treated patients with PD-L1-positive NSCLC, according to results from the phase II POPLAR study and BIRCH trial $[14,15]$.

In $\mathrm{BC}$ the presence of lymphocytic infiltrates as possible prognostic factor was evaluated decades ago. Recently, tumor-infiltrating lymphocytes (TILs) are described as a prognostic factor in Triple Negative Breast Cancer (TNBC). Indeed, Loi et al. demonstrated the association between TILs and better outcomes in this BC subtype evaluating data from the BIG 02-98 trial [16]. In particular, the increment in stromal and intratumoral lymphocytic infiltrations is correlated with the reduction of risk of death. These data were confirmed by Adams et al. that evaluated the correlation between TILs and prognosis in two large phase III clinical trials: ECOG 2197 and ECOG 1199. The authors demonstrated that stromal TILs are an independent prognostic factor for disease free survival (DFS), distant recurrence-free interval (DRFI) and OS [17]. It is important to underline that all these results concern the adjuvant setting and encourage further evaluations to standardize a definition of TILs that could be included in the routine histopathological examination. Confirming the critical role of the immune system in TNBC, an interesting work by Burstein et al. explored TNBC molecular phenotypes using RNA gene expression profiling and described four subtypes of these tumors: Luminal Androgen Receptor (LAR), Mesenchymal (MES), Basal Like Immune-Suppressed (BLIS) and Basal Like Immune-Activated (BLIA). A correlation between these molecular phenotypes and clinical features shows that BLIS subtype has a significantly poorer prognosis in terms of DFS and Disease Specific Survival (DSS) versus all subtypes and conversely the BLIA subtype present the best results in terms of DFS and DSS [18]. These data further support the importance of the immune system in the control of tumor growth and progression. Therefore, Ab anti-CTLA-4, $\mathrm{Ab}$ anti-PD1 and $\mathrm{Ab}$ anti-PDL1 could represent a new effective treatment option in the management of BC. Different studies have demonstrated a positive correlation between CTLA-4, PD-1 and PDL1 with increase in TILs and improved outcomes [19-22]. The checkpoint inhibitors Pembrolimzumab and Atezolizumab were 
evaluated in phase I studies in TNBC [23,24]. In the KEYNOTE-012 trial, approximately $56 \%$ of patients treated with Pembrolizumab experienced a treatment-related adverse event (AE), including fatigue, arthralgia, myalgia, and headache. One patient died of disseminated intravascular coagulation, which was attributed to the immunotherapy. The Overall Response Rate (ORR) was $18.5 \%$ with 1 complete and 4 partial responses. Seven patients had stable disease. Furthermore the phase I trial with Atezolizumab showed that between the 12 patients assessed for safety endpoints, 1 patient experienced grade 3-4 adrenal insufficiency; between the 9 patients assessed for response to therapy, ORR was $33 \%$. Both treatments show activity in this subtype of BC, therefore further trials are investigating these molecules in successive phases of study.

A possible strategy to obtain improved outcomes in terms of DFS and OS is the combination of these immune checkpoint inhibitors with chemotherapy and/or hormonal-therapies. It is worth noting that many chemotherapeutic agents are immunogenic and this could implement the immunotherapeutic effect of checkpoint inhibitors [25]. This concept is reinforced by the fact that chemotherapy is not only immunogenic but also capable to determine significant responses in terms of DFS and OS, responses that could be improved by the combination with immunotherapies [26]. Furthermore, the association between hormonal-therapies and immunotherapies could be active in luminal BC. In this BC subtype the preliminary results of the KEYNOTE-028 trial were presented at the 2015 San Antonio Breast Cancer Symposium [27] and showed activity of Pembrolizumab also in Estrogen receptor positive (ER+)/HER2 negative BCs. Also Avelumab, another $\mathrm{Ab}$ anti-PD-L1, demonstrated to be active in a phase I trial in which responses were observed in all subtypes of BC [28].

Advances in the understanding of the interaction between the immune system and tumor cells have also contributed to the development of other therapeutic strategies based on chimeric antigen receptor (CAR) modified T cells or NK cells. The classic CARs T cells consist of an extracellular antigen recognition domain, a hinge domain, a transmembrane domain and an intracellular domain [29]. The extracellular antigen-binding moiety renders $\mathrm{T}$ cells the ability to bind antigens with retained specificity and affinity [30]. The hinge region mediates CAR flexibility [31] and the transmembrane can indeed influence the function of CAR-T cells [32]. The intracellular domain is responsible for signal delivery within CARs. CAR-T cell immunotherapy demonstrated remarkable clinical responses in hematologic malignancies [33] and now it could represent an interesting challenge in solid tumors treatment. At the same time, given their shorter lifespan and potent cytolytic function, mature NK cells provide attractive candidate effector cells to express CARs and provide an excellent source of therapy for patients with cancer [34].

Another important observation is that the escape from host immunosurveillance resulting in cancer growth and progression depends from different alterations in immune response like the enhancement of immunosuppressive cells such as myeloid-derived suppressor cells (MDSCs). Several approaches to obtain suppression of MDSCs have been sought after [35].

The use of CAR-T or CAR-NK cells and suppression of MDSCs may enhance and sensitize the effects of chemotherapies and checkpoints inhibitors in solid tumors and particularly in BC. Nevertheless more data are still needed to better apply CAR-T, CAR-NK cells and MDSCs techniques in treating solid tumors.
All these data represent the background for a new era in $\mathrm{BC}$ management. As we have seen in our precedent review, combination of different types of treatment is an important strategy to overcome cancer resistance and proliferation. Therefore, combination of new molecules with "classic" therapies, such as chemotherapy and hormonal-therapy, could become the new standard for BC treatment. Further clinical trials are warranted to confirm this interesting hypothesis.

\section{References}

1. Zanardi E, Bregni G, de Braud F, Di Cosimo S (2015) Better Together: Targeted Combination Therapies in Breast Cancer. Semin Oncol 42: 887-895.

2. Hodi FS, O'Day SJ, McDermott DF, Weber RW, Sosman JA, et al. (2010) Improved survival with ipilimumab in patients with metastatic melanoma. N Engl J Med 363: 711-723.

3. Robert C, Thomas L, Bondarenko I, O'Day S, Weber J, et al. (2011) Ipilimumab plus dacarbazine for previously untreated metastatic melanoma. N Engl J Med 364: 2517-2526.

4. Ribas A, Kefford R, Marshall MA, Punt CJ, Haanen JB, et al. (2013) Phase III randomized clinical trial comparing tremelimumab with standard-ofcare chemotherapy in patients with advanced melanoma. J Clin Oncol 31: 616-622.

5. Topalian SL, Sznol M, McDermott DF, Kluger HM, Carvajal RD, et al. (2014) Survival, durable tumor remission, and long-term safety in patients with advanced melanoma receiving nivolumab. J Clin Oncol 32: 1020-1030.

6. Robert C, Ribas A, Wolchok JD, Hodi FS, Hamid O, et al. (2014) Antiprogrammed-death-receptor-1 treatment with pembrolizumab in ipilimumab-refractory advanced melanoma: a randomised dosecomparison cohort of a phase 1 trial. Lancet 384: 1109-1117.

7. Borghaei H, Paz-Ares L, Horn L, Spigel DR, Steins M, et al. (2015) Nivolumab versus Docetaxel in Advanced Nonsquamous Non-Small-Cell Lung Cancer. N Engl J Med 373: 1627-1639.

8. Motzer RJ, Escudier B, McDermott DF, George S, Hammers HJ, et al. (2015) Nivolumab versus Everolimus in Advanced Renal-Cell Carcinoma. N Engl J Med 373: 1803-1813.

9. Krummel MF, Allison JP (1996) CTLA-4 engagement inhibits IL-2 accumulation and cell cycle progression upon activation of resting $\mathrm{T}$ cells. J Exp Med 183: 2533-2540.

10. Waterhouse P, Penninger JM, Timms E, Wakeham A, Shahinian A, et al. (1995) Lymphoproliferative disorders with early lethality in mice deficient in Ctla-4. Science 270: 985-988.

11. Freeman GJ, Long AJ, Iwai Y, Bourque K, Chernova T, et al. (2000) Engagement of the PD-1 immunoinhibitory receptor by a novel B7 family member leads to negative regulation of lymphocyte activation. J Exp Med 192: 1027-1034.

12. Herbst RS, Baas P, Kim DW, Felip E, Pérez-Gracia JL, et al. (2016) Pembrolizumab versus docetaxel for previously treated, PD-L1-positive, advanced non-small-cell lung cancer (KEYNOTE-010): a randomised controlled trial. Lancet 387: 1540-1550.

13. Butte MJ, Keir ME, Phamduy TB, Sharpe AH, Freeman GJ (2007) Programmed death-1 ligand 1 interacts specifically with the B7-1 costimulatory molecule to inhibit $\mathrm{T}$ cell responses. Immunity 27: 111-122.

14. Fehrenbacher L, Spira A, Ballinger M, Kowanetz M, Vansteenkiste J, et al. (2016) Atezolizumab versus docetaxel for patients with previously treated non-small-cell lung cancer (POPLAR): a multicentre, open-label, phase 2 randomised controlled trial. Lancet 387: 1837-1846.

15. Besse B (2015) Phase II trial (BIRCH) of atezolizumab as first-line or subsequent therapy for advanced PD-L1-selected non-small cell lung cancer (NSCLC). European Cancer Congress.

16. Loi S, Sirtaine N, Piette F, Salgado R, Viale G, et al. (2013) Prognostic and predictive value of tumor-infiltrating lymphocytes in a phase III 
Citation: Zanardi E, Di Cosimo S (2016) Better Together: Immunotherapy as Future Combination Strategy for Breast Cancer. Immunother Open Acc 2: 120. doi:10.4172/2471-9552.1000120

Page 3 of 3

randomized adjuvant breast cancer trial in node-positive breast cancer comparing the addition of docetaxel to doxorubicin with doxorubicinbased chemotherapy: BIG 02-98. J Clin Oncol 31: 860-867.

17. Adams S, Gray RJ, Demaria S, Goldstein L, Perez EA, et al. (2014) Prognostic value of tumor-infiltrating lymphocytes in triple-negative breast cancers from two phase III randomized adjuvant breast cancer trials: ECOG 2197 and ECOG 1199. J Clin Oncol 32: 2959-2966.

18. Burstein MD, Tsimelzon A, Poage GM, Covington KR, Contreras A, et al. (2015) Comprehensive genomic analysis identifies novel subtypes and targets of triple-negative breast cancer. Clin Cancer Res 21: 1688-1698.

19. Denkert C, von Minckwitz G, Brase JC, Sinn BV, Gade S, et al. (2015) Tumor-infiltrating lymphocytes and response to neoadjuvant chemotherapy with or without carboplatin in human epidermal growth factor receptor 2-positive and triple-negative primary breast cancers. J Clin Oncol 33: 983-991.

20. Schalper KA, Velcheti V, Carvajal D, Wimberly H, Brown J, et al. (2014) In situ tumor PD-L1 mRNA expression is associated with increased TILs and better outcome in breast carcinomas. Clin Cancer Res 20: 2773-2782.

21. West NR, Kost SE, Martin SD, Milne K, Deleeuw RJ, et al. (2013) Tumour infiltrating FOXP3(+) lymphocytes are associated with cytotoxic immune responses and good clinical outcome in oestrogen receptor-negative breast cancer. Br J Cancer 108: 155-162.

22. Loi S, Michiels S, Salgado R, Sirtaine N, Jose V, et al. (2014) Tumor infiltrating lymphocytes are prognostic in triple negative breast cancer and predictive for trastuzumab benefit in early breast cancer: results from the FinHER trial. Ann Oncol 25: 1544-1550.

23. Nanda R, Chow LQ, Dees EC, Berger R, Gupta S, et al. (2015) A phase Ib study of pembrolizumab (MK-3475) in patients with advanced triple negative breast cancer. Clin Cancer Res 75: S1-09.

24. Emens LA, Braiteh FS, Cassier P, DeLord JP, Eder JP, et al. (2015) Inhibition of PD-L1 by MPDL3280A leads to clinical activity in patients with metastatic triple negative breast cancer. Clin Cancer Res 75: PD1-6.

25. Galluzzi L, Senovilla L, Zitvogel L, Kroemer G (2012) The secret ally: immunostimulation by anticancer drugs. Nat Rev Drug Discov 11: $215-233$.
26. Ribas A, Hersey P, Middleton MR, Gogas H, Flaherty KT, et al. (2012) New challenges in endpoints for drug development in advanced melanoma. Clin Cancer Res 18: 336-341.

27. Rugo HS, Delord J-P, Im SA (2015) Preliminary efficacy and safety of pembrolizumab (MK-3475) in patients with PD-L1-positive, estrogen receptor-positive $(\mathrm{ER}+) / \mathrm{HER} 2$-negative advanced breast cancer enrolled in KEYNOTE-028.

28. Dirix LY, Takacs I, Nikolinakos P (2015) Avelumab (MSB0010718C), an anti-PD-L1 antibody, in patients with locally advanced or metastatic breast cancer: A phase Ib JAVELIN solid tumor trial. Presented at: San Antonio Breast Cancer Symposium.

29. Whilding LM, Maher J (2015) CAR T-cell immunotherapy: The path from the by-road to the freeway? Mol Oncol 9: 1994-2018.

30. Shi H, Sun M, Liu L1, Wang Z (2014) Chimeric antigen receptor for adoptive immunotherapy of cancer: latest research and future prospects. Mol Cancer 13: 219.

31. Hudecek M, Sommermeyer D, Kosasih PL, Silva-Benedict A, Liu L, et al. (2015) The non-signaling extracellular spacer domain of chimeric antigen receptors is decisive for in vivo antitumor activity. Cancer Immunol Res 3: 125-135.

32. Dotti G, Gottschalk S, Savoldo B, Brenner MK (2014) Design and development of therapies using chimeric antigen receptor-expressing $\mathrm{T}$ cells. Immunol Rev 257: 107-126.

33. Maude SL, Frey N, Shaw PA, Aplenc R, Barrett DM, et al. (2014) Chimeric antigen receptor $\mathrm{T}$ cells for sustained remissions in leukemia. $\mathrm{N}$ Engl J Med 371: 1507-1517.

34. Rezvani K, Rouce RH (2015) The Application of Natural Killer Cell Immunotherapy for the Treatment of Cancer. Front Immunol 6: 578.

35. Katoh H, Watanabe M (2015) Myeloid-Derived Suppressor Cells and Therapeutic Strategies in Cancer. Mediators Inflamm 2015: 159269. 